\title{
Experimental realization of a topological Anderson insulator
}

\author{
S. Stützer ${ }^{1}$, M. C. Rechtsman ${ }^{2}$, P. Titum ${ }^{3}$, Y. Plotnik ${ }^{2}$, Y. Lumer ${ }^{2}$, J. M. \\ Zeuner $^{1}$, S. Nolte ${ }^{1}$, G. Refael ${ }^{3}$, N. Lindner ${ }^{2}$, M. Segev ${ }^{2}$, and A. Szameit ${ }^{1}$ \\ ${ }^{1}$ Institute of Applied Physics, Abbe Center of Photonics, Friedrich-Schiller-Universität Jena, \\ Max-Wien-Platz 1, 07743 Jena, Germany \\ ${ }^{2}$ Department of Physics, Technion - Israel Institute of Technology, Haifa 32000, Israel \\ ${ }^{3}$ Institute of Quantum Information and Matter, Dept. of Physics, Caltech, Pasadena, CA 91125
}

\begin{abstract}
We experimentally demonstrate that disorder can induce a topologically non-trivial phase. We implement this ,Topological Anderson Insulator“ in arrays of evanescently coupled waveguides and demonstrate its unique features.

(c) 2014 Optical Society of America

OCIS codes: (240.0240) Optics at surfaces; (080.1238) Array waveguide devices
\end{abstract}

Topological states have experienced ongoing fascination in condensed matter physics, and in particular the discovery of topological insulators [1] was one of the undisputed highlights of recent years. Topological protection in solids, that is, the scatter-free evolution of an edge current, is commonly achieved either by strong magnetic fields, i.e., the quantum Hall effect, or by spin-orbit coupling in topological insulators. Following the progress in condensed matter, where topologic insulators rely on electronic properties such as spin-orbit coupling, much effort was dedicated to introduce the ideas into photonics. At low frequencies (microwaves), photonic topological insulators were indeed demonstrated - where they rely on magnetic (gyro-optic) effects [2]. But in the domain of optics, magnetic effects are extremely weak and spin-orbit effects, being fundamentally different than that of electrons (fermions), do not lead to immunity against backscattering. Hence, realizing photonic topological insulators required a new paradigm. Various avenues were suggested $[3,4,5]$ until eventually two different ideas led to the experimental demonstration of photonic topological insulators in the optical regime: [6] and [7]. The first one [6], realized by our team, relies on the so-called Floquet topological insulators $[8,9]$ : periodically-modulated photonic graphene. This is a 2D lattice of evanescently coupled waveguides that are arranged in a honeycomb (graphene-like) geometry, where each waveguide is twisted around its axis in a helical fashion, similar to a single strand of DNA (see Fig. 1(a)). Such a system can be described by the paraxial, Schrödinger-type, wave equation:

$$
i \partial_{z} \psi=-\frac{1}{2 k}\left(i \nabla_{\perp}-A(z)\right)^{2} \psi-\frac{k \Delta n(x, y)}{n_{0}} \psi,
$$

where $\psi$ is the envelope function of the EM field, $k$ is the wavenumber of light in the medium, $n_{0}$ is the refractive index of the medium, and $\Delta n(x, y)$ is the refractive index change. In a frame of reference moving with the helicity of the waveguides, we find that the gauge field $A(z)=(\cos (\Omega z), \sin (\Omega z))$ acts to breaks the $z$-reversal symmetry.
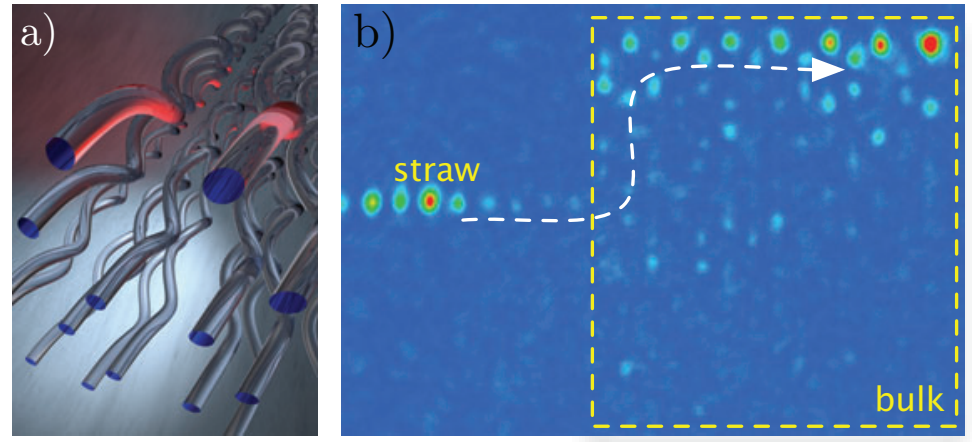

Figure 1. a) Sketch of a photonic lattice with a graphene-like structure and twisted waveguides. This system acts as a topological insulator. b) The wave function at the output clearly travelled along the edge without any scattering.

In what follows, we demonstrate a system where disorder makes it topological, leading to unidirectional topologically protected edge states by virtue of disorder.

Since 1958, it has been unequivocally accepted that disordered systems exhibit Anderson localization: the suppression of waves transport due to multiple scattering from disorder. In fact, in two-dimensional disordered systems, all eigenstates are localized even for an infinitesimal amount of randomness, without any observable threshold. In contrast, 
the radically different nature of topological systems allows them to retain extended states even under the influence of disorder in two dimensions. However, it was still extremely surprising when the so-called "Topological Anderson Insulator" was proposed [10]: a system which is transformed into being topological - displaying extended edge modes - by the presence of disorder. Conceptually, in the absence of disorder, such a system exhibits a trivial bandgap co-existing with broken time-reversal symmetry [10]. Prior to this current work, Topological Anderson Insulators have not been demonstrated in any experiment in any system in nature.

Here, we present the first experimental demonstration of a Topological Anderson Insulator. We use a photonic system similar to our work in [6]. However, here we employ a staggered mass potential, that is, the two sublattices of the honeycomb structure exhibit different refractive indices $n_{a}$ and $n_{b}$ (see Fig. 2 a)). When $n_{a}=n_{b}$ our system is identical to the FTI in [6] and, hence, exhibits topologically protected edge states (Fig. 1 b)). However, when breaking the inversion symmetry in the transverse plane by detuning the sublattices $\left(n_{a} \neq n_{b}\right)$ a topologically trivial bandgap opens and topological insulation vanishes. This trivial bandgap closes in the presence of disorder (helically synchronized with the lattice) and acquires a nonzero topological invariant when it finally reopens. This is impressively demonstrated by the formation of a one-way edge state, as shown in Fig. 2 b).

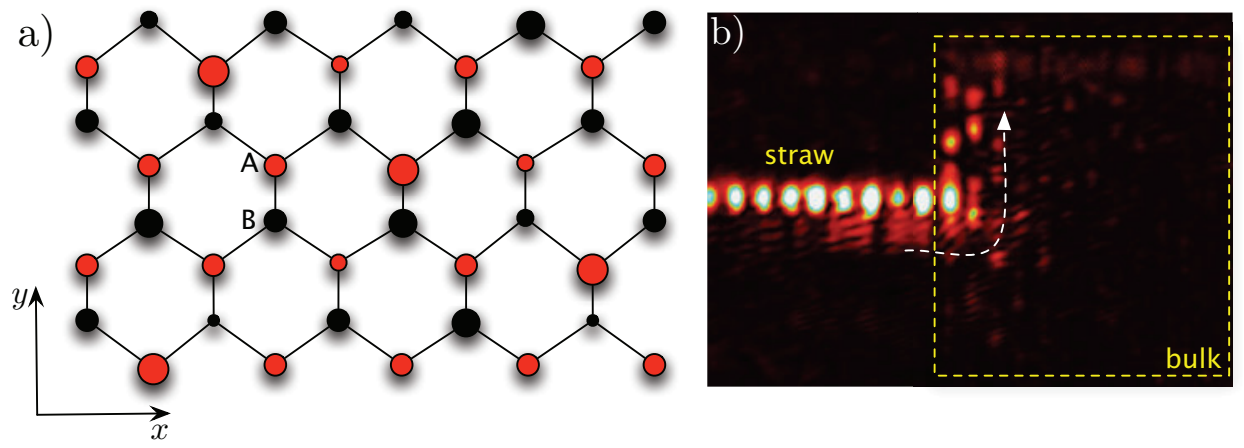

Figure 2. a) Schematic representation of the honeycomb lattice with a staggered mass potential composed of two sublattices A (red) and B (black). The uniformly disordered onsite energy is indicated by variable radius of the lattice sites. b) Wave packet at the sample output. The observed edge state in the disordered system is clearly visible.

The fundamental concept of our TAI is as follows. In the periodically driven system with a staggered potential the drive (the helicity manifested in the gauge $A(z)$ ) and staggering act as competitors. The staggering produces a trivial gap with positive effective mass at both Dirac cones, whereas the drive causes a topological gap with opposite masses. When the staggering dominates over the drive, the system has trivial topology. This is where disorder comes in: it effectively reduces the effect of the staggering and, at sufficiently large disorder it closes the trivial gap and the topological phase is restored.

For our experiments, we fabricate waveguide arrays in fused silica by direct femtosecond laser writing [11]. By tuning the position and velocity of the laser spot during the writing process with highest precision, we design samples with controlled staggering, disorder and geometrical properties. Which allows us to observe the extended TAI edge modes (Fig. 2 b)).

In conclusion, we presented the first experimental realization of the Topological Anderson Insulator, where disorder drives the system into a topologically non-trivial phase.

\section{References}

1. Phys. Rev. Lett. 95, 226801 (2005).

2. Nature 461, 772-775 (2009).

3. Phys. Rev. A 84, 043804 (2011).

4. Nature Physics 7, 907-912 (2011).

5. Nature Materials 12, 233-239 (2013).

6. Nature 496, 196-200 (2013).

7. Nature Photonics 7, 1001-1005 (2013).

8. Nature Physics 7, 490-495 (2011).

9. Phys. Rev. B 82, 235114 (2010).

10. Phys. Rev. Lett. 102, 136806 (2009).

11. J. Phys. B 43, 163001(2010). 